\title{
AN UPDATE ON HEPATITIS C INFECTION AND LOCAL MANAGEMENT
}

\author{
D Das, Specialist Registrar; C Brown, Consultant Physician; \\ D Telford, Consultant Microbiologist; Lancaster and Kendal Hospitals. \\ P Morgan-Capner, Public Health Laboratory Service, North West
}

\section{INTRODUCTION}

This paper is an update on hepatitis $\mathrm{C}$ virus (HCV) infection and is based on a mini-symposium offered at Lancaster Postgraduate Medical Centre in January 1998. Viral biology, epidemiology and clinical effects are discussed, together with the merits and drawbacks of currently recommended therapies and aspects of local service provision.

Since the early 1970 s when testing for hepatitis A and B became possible, it was apparent that there was at least one further transmissible agent causing non-A - non-B hepatitis, particularly in post-transfusion cases. The virus was cloned by molecular techniques from the DNA extracted from infected chimpanzees, leading to the development of antibody tests in 1989. The enzyme immunoassays for antibodies were refined and routine blood donor screening was introduced in 1991.

There is a highly conserved RNA region of the viral genome which has facilitated development of the enzyme immunoassay tests. There is also a much more variable region, which has given rise to defining six genotypes or quasispecies. Type 1a is commoner in northern Europe and the UK, whilst type 3 is associated with intravenous drug abuse. The variability of the viral RNA genome allows mutation under 'immune pressure' and escape from neutralising antibodies. This leads to viral persistence and chronic inflammation and poses a major challenge for the development of a successful vaccine.

\section{EPIDEMIOLOGY AND MODE OF TRANSMISSION}

There are approximately 170 million people with HCV infection worldwide, with particularly high carriage rates in some countries, such as Egypt and Rwanda $(>10 \%)$. The prevalence rate varies considerably but of countries with reliable health statistics, the UK has the lowest prevalence with around 200,000 cases in total. The 'blood transfusion lookback study' identified approximately 800 cases of posttransfusion hepatitis $\mathrm{C}$ infection, giving a total estimate of $9,000-12,000$ infected subjects in the northwest region. Amongst blood donors, $0.04 \%$ were infected with $\mathrm{HCV}$, of which $51 \%$ were attributed to intravenous drug abuse, $20 \%$ to transfused blood products and the source uncertain in the remainder. It is unclear why the process of dispensing sterile needles has successfully contained the spread of HIV infection but not that of $\mathrm{HCV}$.

The risk of transmitting $\mathrm{HCV}$ by other routes, including accidental sharps injuries and human bites, is minimal (1$3 \%$ ). Sexual transmission can occur but the risk is low and testing of sexual partners has to be considered on an individual basis. Sexual behaviour need not be adjusted in longterm relationships, but some clinicians suggest the use of condoms with new partners. Vertical transmission can also occur from mother to neonate, but the risk is low (1-6\%) and associated with high viral load or co-infection with HIV virus, but transmission through breast milk does not seem to occur.

Data from diagnostic serological testing in the northwest region (Public Health Laboratory Service April-September 1997) has revealed 970 positive cases out of 6,305 patients tested, with a high prevalence occurring in Liverpool and in HM prisons. There were 61 cases in Morecambe Bay in the same period.

\begin{tabular}{|c|c|c|}
\hline AREA & $\begin{array}{c}\text { NUMBER OF } \\
\text { SAMPLES TESTED }\end{array}$ & $\begin{array}{c}\text { NUMBER OF } \\
\text { SAMPLES POSITIVE }\end{array}$ \\
\hline Preston & 458 & 27 \\
\hline Morecambe Bay & 363 & 61 \\
\hline Liverpool & 998 & 349 \\
\hline Chester & 80 & 8 \\
\hline HM Prisons & 204 & 78 \\
\hline
\end{tabular}

Table 1 Hepatitis $C$ - the local scene

\section{NATURAL HISTORY AND DISEASE PROGRESSION}

Acute infection with HCV is very rarely encountered due to lack of specific symptoms and minor abnormalities in liver function tests. In $85 \%$ of cases, acute infection leads to chronic persistence of the virus and $60 \%$ of this group of carriers ultimately develop chronic hepatitis. Approximately $20 \%$ of those with chronic hepatitis will progress to cirrhosis, usually over 10 to 20 years, and amongst those with cirrhosis approximately $1-5 \%$ per annum develop hepatocellular carcinoma. There is considerable variability in the length of time of progression to cirrhosis and liver cancer. Younger subjects fare better than older ones. Co-infection with hepatitis B or significant alcohol abuse accelerate the disease process but only a small minority of cases reach a critical point where longevity is compromised.

\section{TESTING FOR HCV INFECTION}

Three groups of patients should be considered for HCV screening: firstly, those with an unexplained and persistent (>3 months) rise in serum ALT, irrespective of risk factors; secondly, active or reformed drug abusers; thirdly, patients presenting with 'cryptogenic' chronic liver disease. The PHLS offers a screening antibody test (enzyme immunoassay, EIA-3) and positive cases are confirmed using a more 
specific radio-immunoblot assay (RIBA-3). Detection of circulating virus in the bloodstream requires an expensive polymerase chain reaction technique (PCR) which costs $£ 70$ and should be reserved only for those patients being considered for interferon therapy, or monitoring treatment efficacy. Quantitative measurements of viral load and viral genotyping are currently research tools.

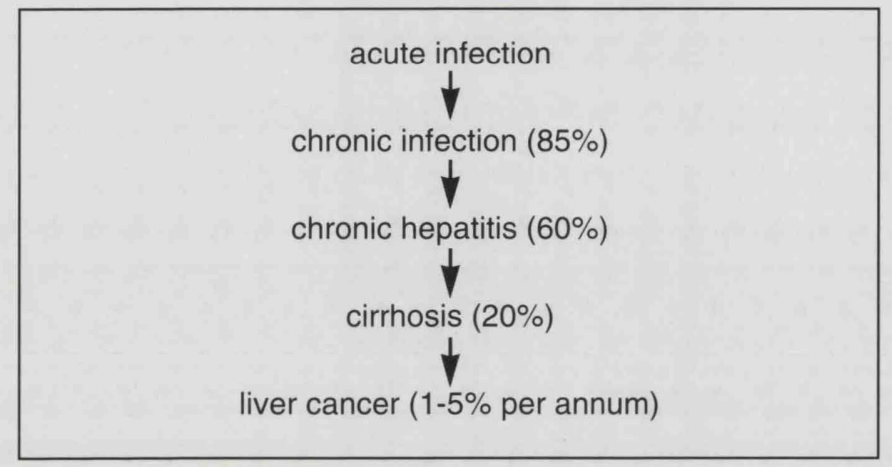

Figure I Algorithm of HCV infection

\section{INTERFERON THERAPY}

Alpha-interferon is currently the only licensed treatment for hepatitis $\mathrm{C}$ infection in the UK. Interferon prevents viral attachment to cells and reduces intracellular replication. Expression of viral antigens on cell membranes is enhanced, promoting cell destruction by circulating cytotoxic $\mathrm{T}$ cells.

Interferon is injected subcutaneously in a dose of three mega units three times a week for 12 months. Treatment efficacy is assessed by PCR testing for active viraemia two months into a treatment course. Viral persistence at this stage indicates treatment failure and treatment can be stopped at the next clinic visit. A negative PCR test at this stage prompts completion of a twelve-month treatment course. Successful elimination of HCV following this treatment occurs in 20$30 \%$ of cases, although higher clearance occurs with higher doses and with co-administration with an oral nucleoside called ribavarin, which is unlicensed at present.

Limited efficacy is not the only problem associated with interferon therapy. It is also expensive ( $£ 2,500$ per treatment) and has a high incidence of side effects. One gram of paracetomol taken one hour prior to dosing with interferon minimises troublesome flu-like symptoms. Bone marrow depression is dose-related, usually occurring in the first few weeks and some patients can become psychologically depressed. Autoimmune diseases such as thyroiditis and diabetes mellitus can be provoked by interferon and chronic autoimmune hepatitis coincidental with HCV infection can be exacerbated by this treatment.

Interferon therapy is indicated when active $\mathrm{HCV}$ viraemia is associated with significant chronic inflammation in a liver biopsy, graded in an internationally recognised scoring system (Ishak score of six or more). It is worth noting that the overall mortality associated with a percutaneous liver biopsy is about one in 500 although this figure is skewed by patients with liver cancer and coagulopathy. Some contraindications for interferon therapy (and hence PCR testing and liver biopsy) are listed in Table 2, although children may be treated in specialist tertiary centres.

Adequate patient selection is required in view of limited efficacy and side effects of treatment, restricted patient compliance and high costs of treatment. Decisions to start treatment are made on the merits of each case but there are some general exclusions (Table 2).
Age $<18$ years or $>65$ years

Pregnancy

Alcohol abuse

Poor attendance/compliance

Depression/psychosis

Currently on intravenous drugs/methadone

Severe comorbid disease

HM prison resident

\section{Table 2 Contraindications to interferon treatment}

Patients who continue to abuse intravenous drugs are at risk of septicaemia and thrombo-embolic disease with little chance of interferon significantly influencing longevity. Patients on methadone replacement therapy may be regarded as being in an interim phase and treatment may be deferred for those who subsequently rehabilitate successfully and is not necessarily provided for those who relapse their drug habit. A decision to defer treatment in those taking methadone can be justified in view of the length of time it takes for chronic hepatitis to progress to cirrhosis.

\section{HEPATITIS C SERVICES IN MORECAMBE BAY}

Local organisation and provision of services for patients with $\mathrm{HCV}$ infection is shared between primary and secondary care, with support from tertiary services such as the PHLS laboratory service at Preston and occasionally the regional liver units.

At the community level, most cases of HCV infection are detected by general practitioners and medical officers for local prisons, drug rehabilitation units and STD clinics. Intravenous drug abusers and patients with unexplained increases in liver transaminases are screened for HCV virus.

There are three consultant physicians within Morecambe Bay to whom patients with HCV infection may be referred for further management: Drs C Brown, J Keating and A Higham. Counselling and providing information to patients is important, particularly regarding disease outcomes, lifestyle advice (drugs and alcohol), risks and merits of liver biopsy and interferon treatment. Testing for active $\mathrm{HCV}$ viraemia (PCR positive) as a prelude to liver biopsy if positive, should be reserved for patients considered suitable for interferon treatment. Physicians are required to monitor those patients on treatment for drug efficacy and side effects, withdrawing therapy from treatment failures at an early stage (two to three months). Those completing a 12-month treatment course require PCR testing and a liver biopsy to assess response. The key decision in determining need for therapy is interpretation of the degree of necro-inflammatory activity in a liver biopsy by our three consultant histopathologists, with a threshold score of six or more on an Ishak scoring system (Figures 2 and 3). Other key personnel include radiologists, pharmacists and nursing staff.

There are many uncertain areas in HCV management and a flexible approach is therefore required. Those with mild changes in liver biopsy are currently kept under review pending a possible repeat liver biopsy in two to three years. Funding is an area of diffculty, particularly as Wessex region considered treatment in HCV infection to have "an unproven benefit" in 1993. Health authorities have been reluctant to provide explicit support, but this approach does not make this 


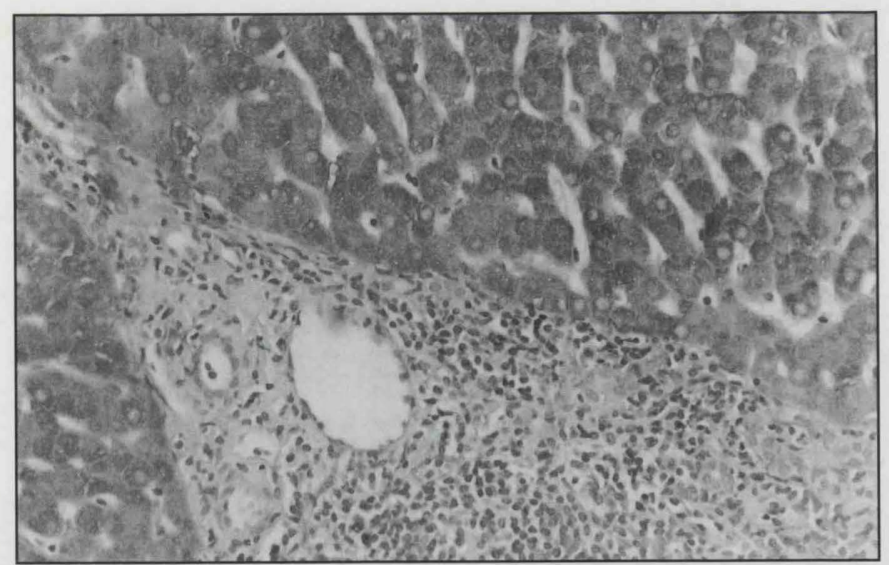

Figure 2 There is an infiltrate of lymphocytes in the portal tract but there is no interface hepatitis (piecemeal necrosis), ie the outline of the hepatocyte islands is well-defined and there is no hepatocyte loss.

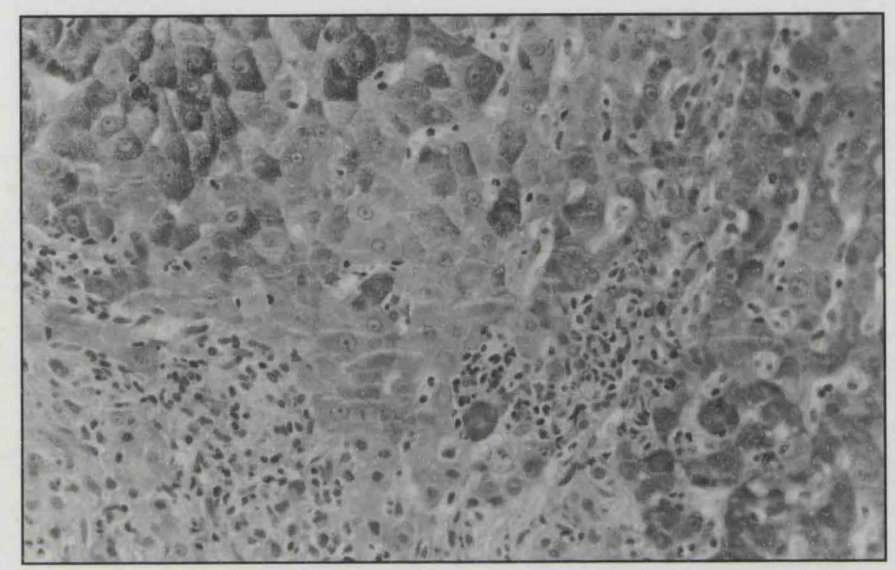

Figure 3 This shows an infiltration of lymphocytes in the portal tract with interface hepatitis, ie the lymphocytes infiltrate between the hepatocytes with cell destruction and irregularity of the outline. The presence of interface hepatitis increases the Ishak score.

difficult area go away! A practical approach has been adopted in Morecambe Bay, with careful and judicious screening of patients with $\mathrm{HCV}$ infection, to try to focus therapy on those patients who may benefit from treatment.

\section{SUMMARY}

In conclusion, this article has focused on the hepatitis $\mathrm{C}$ virus and its clinical effects and outcomes. It has also illustrated the diffculties relating to patient assessment, liver biopsy and the merits and drawbacks of interferon therapy. This is one evolving area in clinical gastroenterology, but whilst trying to shed light on some grey areas, this review hopefully shows that management of $\mathrm{HCV}$ infection is far from black and white!

\section{REFERENCES}

1 Sherlock S Clinical features of hepatitis. In Zuckemman AJ, Thomas HC (eds) Viral Hepatitis: Scientific Basis and Clinical Management. Edinburgh, Churchill Livingstone 1993 pp 1-17

2 De Medina M, Schiff ER Hepatitis C: diagnostic assays. Semin Liver Dis 1995;15:33-40

3 Simmonds P, Smith DB, McOmish F et al Identification of genotypes of hepatitis $\mathrm{C}$ virus sequence comparisons in the core, E1 and NS-5 regions J Gen Virol 1994;75:1053-1061

4 Zein NN, Rakela J, Krawitt EL et al Hepatitis C virus genotypes in the United States: epidemiology, pathogenicity and response to interferon therapy Ann Intern Med 1996; $125: 634-639$

5 Gretch DR Diagnostic tests for hepatitis C. Presented at the NIH Consensus Conference on Management of Hepatitis C March 24-26 1997 Washington DC

6 Ishak K, Baptista A, Bianchi L et al Histological grading and staging of chronic hepatitis J Hepatol 1995;22:696-699

7 Gretch DR, Rosa CD, Carithers RL et al Assessment of hepatitis $\mathrm{C}$ viraemia using molecular amplification technologies: correlation and clinical implications J Hepatol $1995 ; 23: 509-518$

8 Booth J, Foster G, Kumar U et al Chronic hepatitis C virus infection; predictive value of genotype and level of viraemia on disease progression and response to interferon alpha Gut $1995 ; 36: 427-432$

9 Poynard T, Bedossa P, Chevalier M et al A comparison of three interferon alpha $2 \mathrm{~b}$ regimens for the longterm treatment of chronic non-A, non-B hepatitis NEJM 1995;332:14571462 